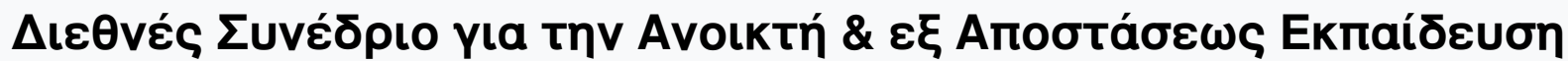

Tóp. 7, Ap. 5B (2013)

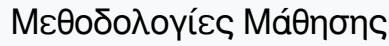

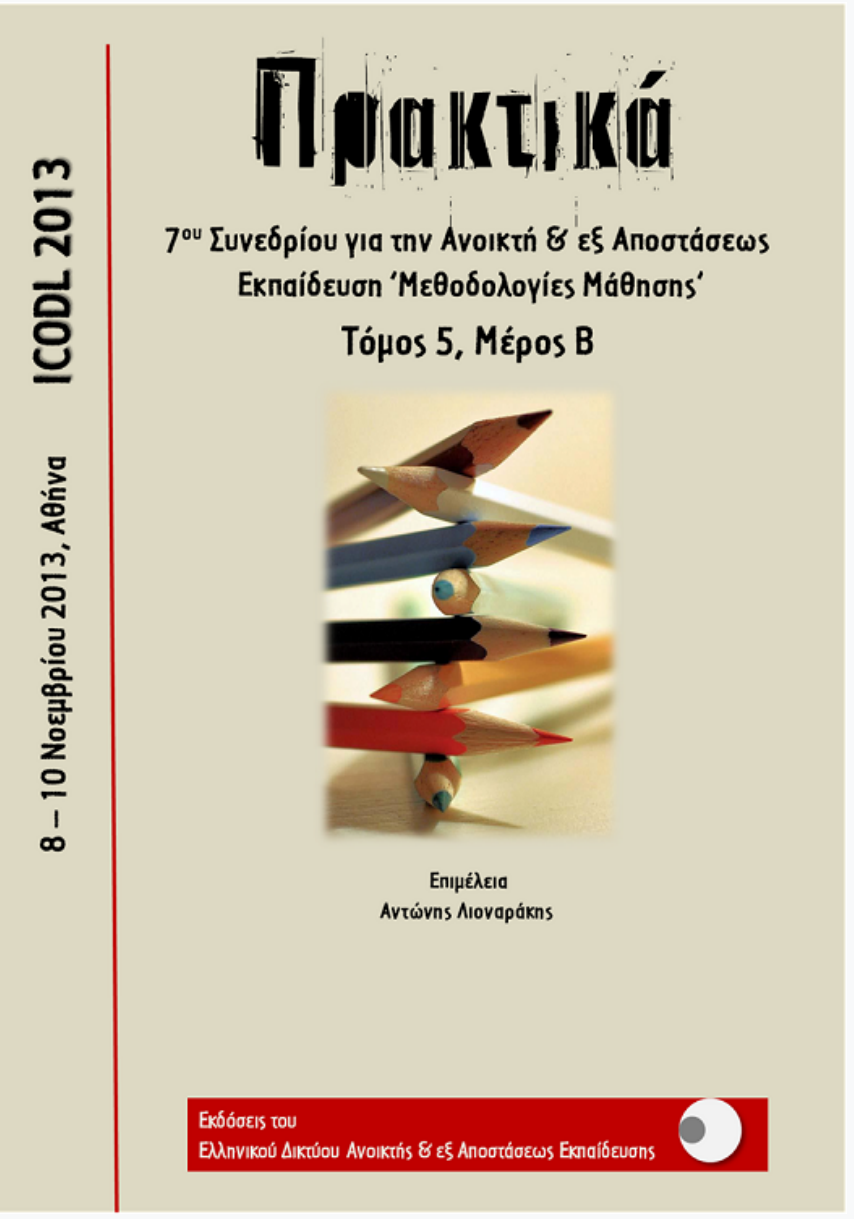

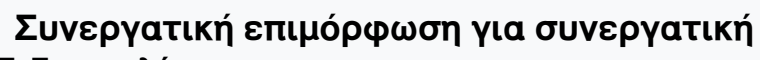

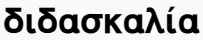

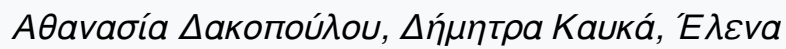
Maviátn

doi: $10.12681 /$ icodl. 554 


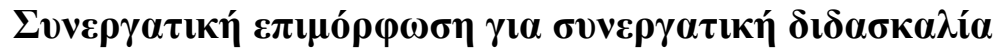

\section{Collaborative in-service teacher training for collaborative teaching}

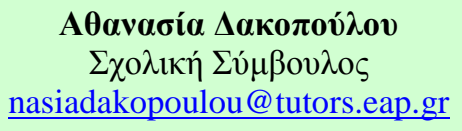

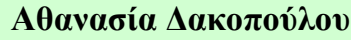

$\Sigma \chi 0 \lambda 1 \kappa \eta ́ ~ \Sigma u ́ \mu \beta o v \lambda o \varsigma$

nasiadakopoulou@tutors.eap.gr

\author{
$\Delta \eta \dot{\mu \eta \tau \tau \alpha ~ К \alpha v \kappa \alpha ́ ~}$

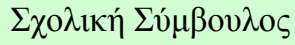 \\ dimitrakauka@gmail.com
}

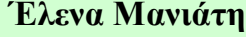 \\ $\Sigma \chi 0 \lambda 1 \kappa \eta ́ ~ \Sigma u ́ \mu \beta o v \lambda o s$ \\ elmaniati@gmail.com
}

\begin{abstract}
The dramatic developments that modern societies experience undoubtedly impinge upon education and the teaching/learning process. In particular, the multifaceted diversity of the student population and the concomitant need to take into account a host of variables necessitate the exploration of alternative approaches to teaching and urge the adoption of transformative action towards an all-inclusive education.

As a result, contemporary forms of in-service teacher training promote alternative teaching practices to accommodate students' differential needs. To this end, the adoption of collaborative dispositions and attitudes on the part of the teachers is valuable.

From this vantage point, this paper presents an innovative learning process in lifelong learning and education. In short, it is a participatory and collaborative educational technique applicable both to school and adult education usually referred as "jigsaw".

The paper focuses on an experiential learning action and in particular the simulation of "jigsaw" to primary school teachers which took place in three educational primary school districts in Attica during 2012 with the participation of teachers from 32 school units.

The paper will describe the key points of the action, the benefits and potential difficulties and the way to overcome them and provide some critical comments towards its implementation. Finally, it will expose the preconditions of its effective implementation.
\end{abstract}

Keywords: in-service teacher training, collaborative teaching, jigsaw

\section{Пєрí $\lambda \eta \psi \eta$}

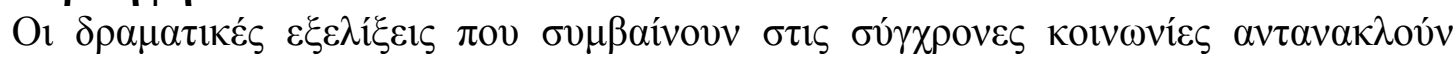

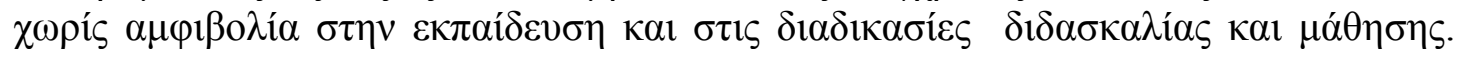

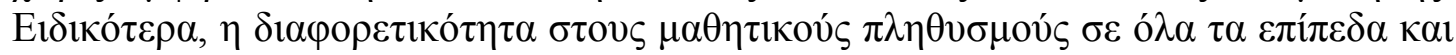

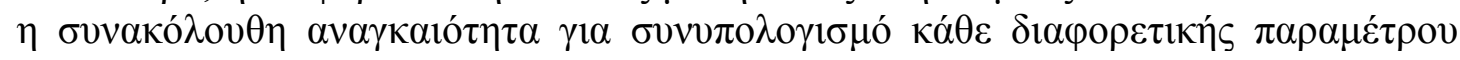

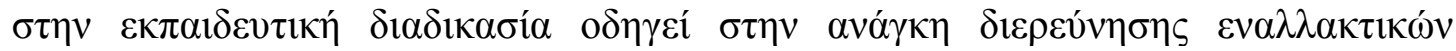

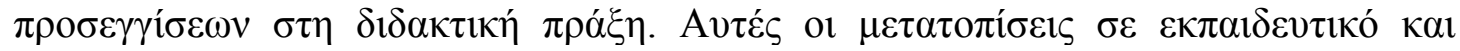

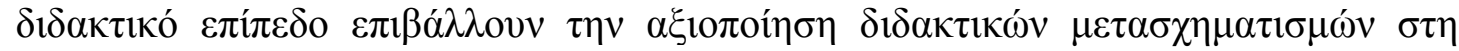

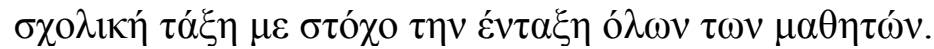

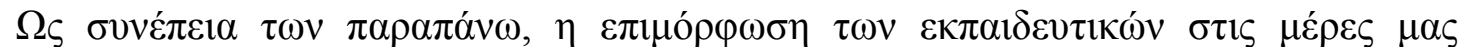

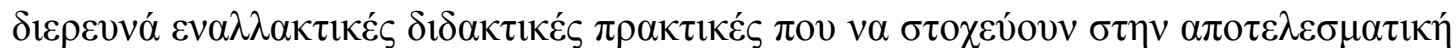

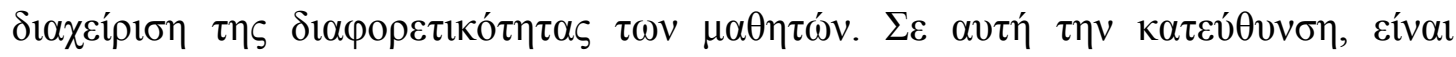




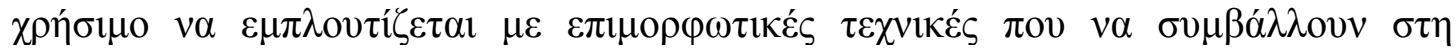

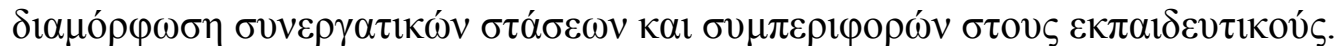

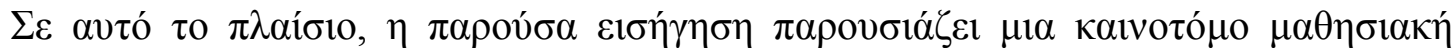

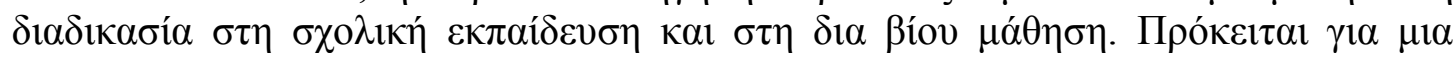

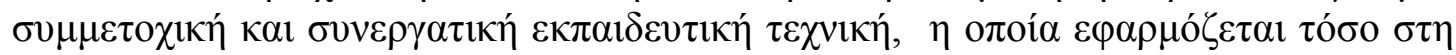

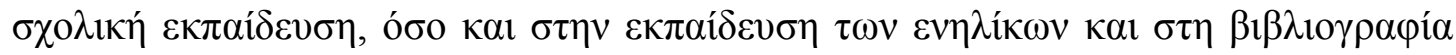

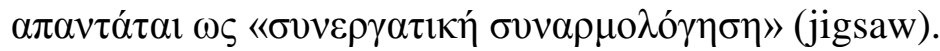

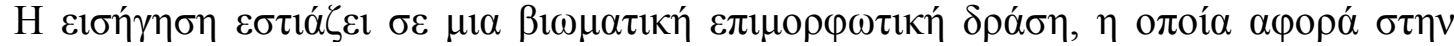

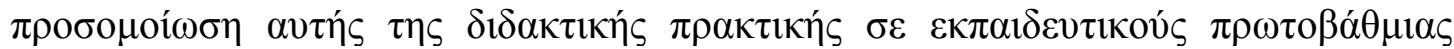

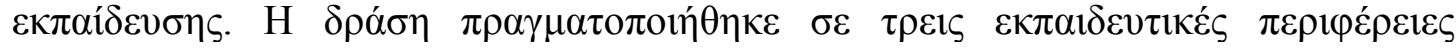

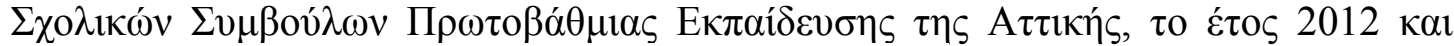

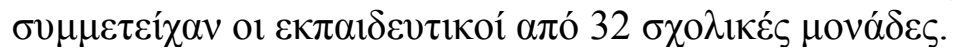

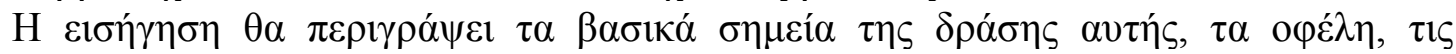

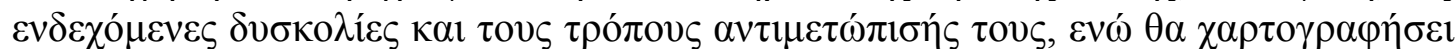

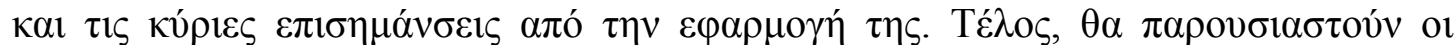

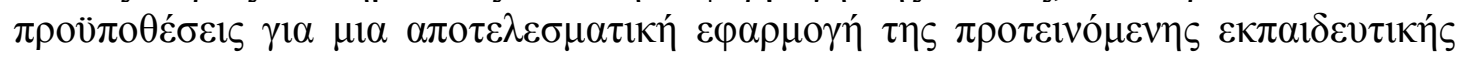

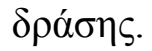

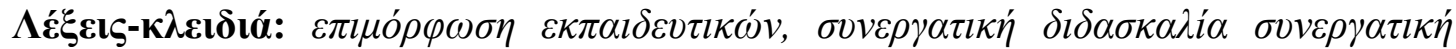

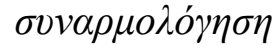

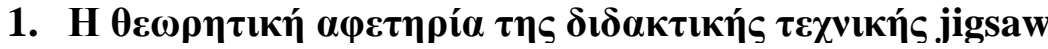

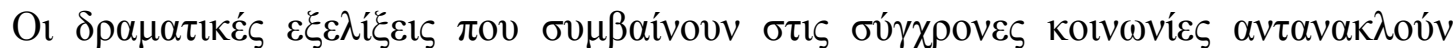

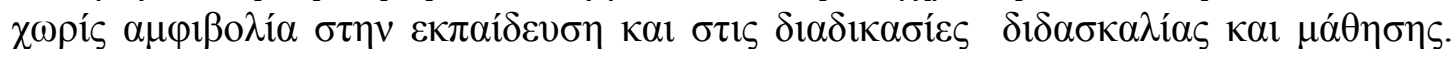

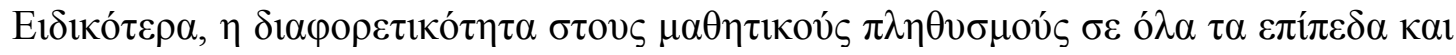

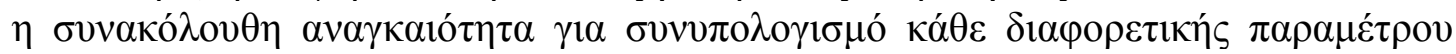

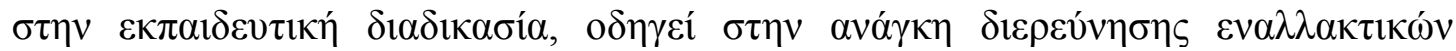

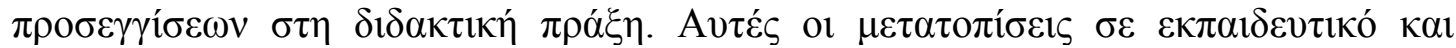

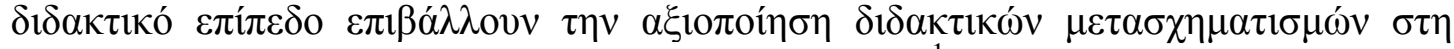

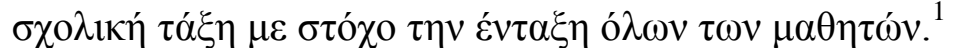

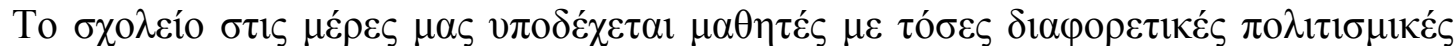

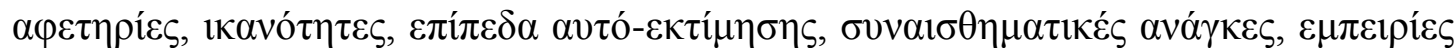

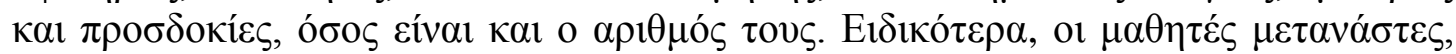

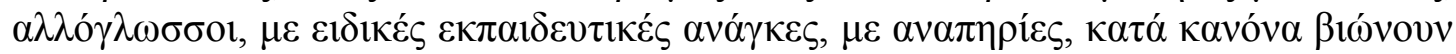

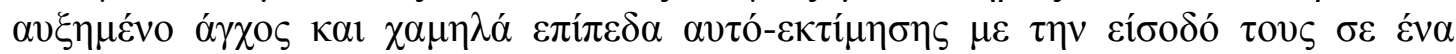

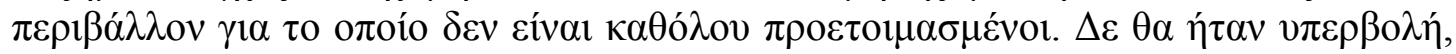

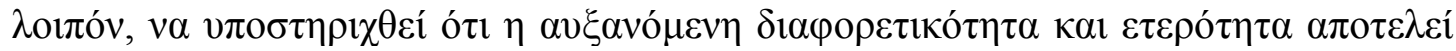

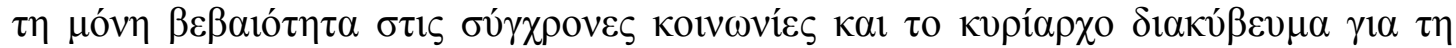

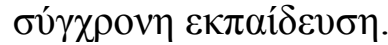

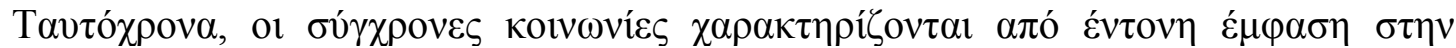

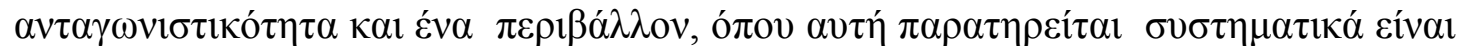

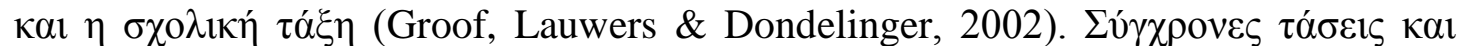

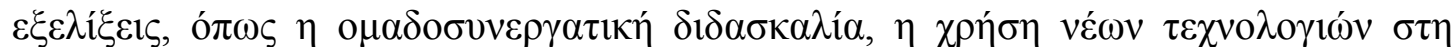

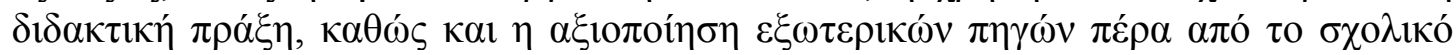

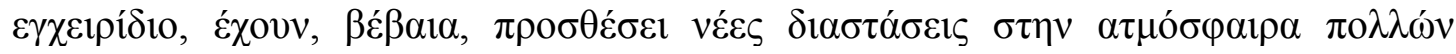

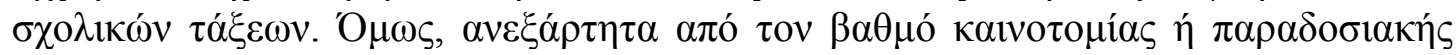

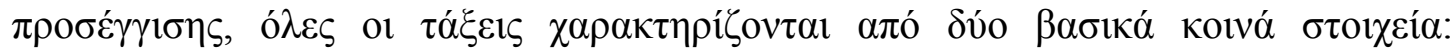

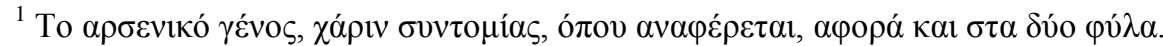

SECTION B: applications, experiences, good practices, descriptions and outlines, educational activities, issues for dialog and discussion
} 


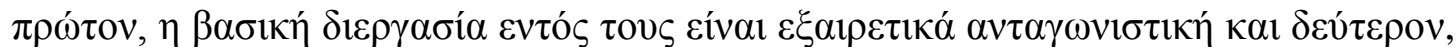

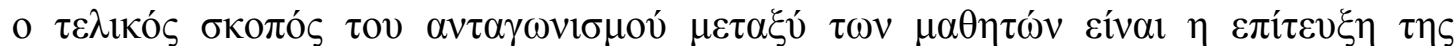

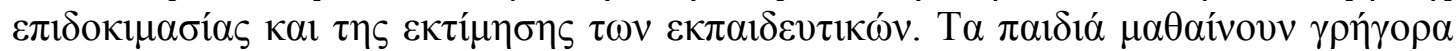

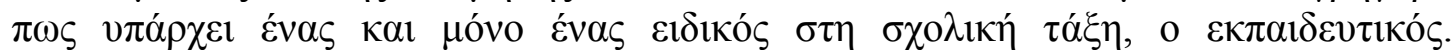

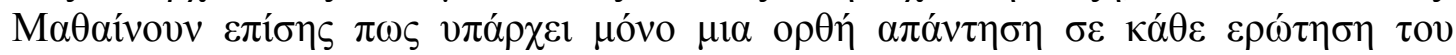

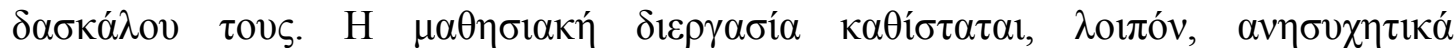

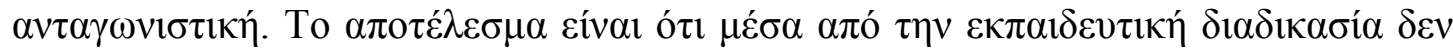

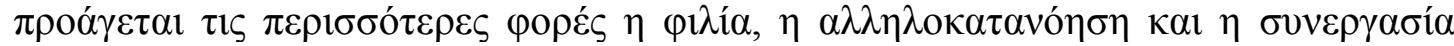

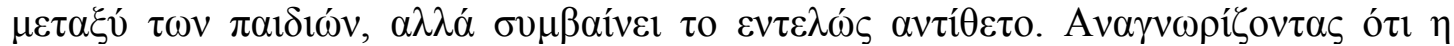

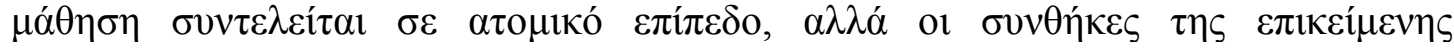

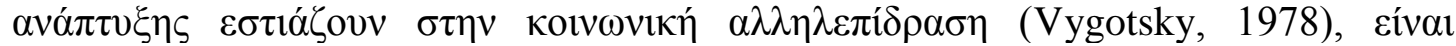

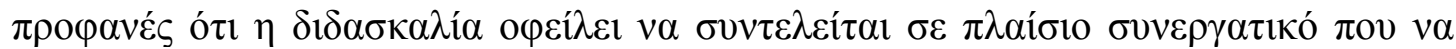

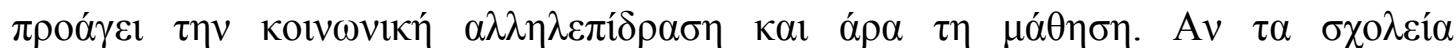

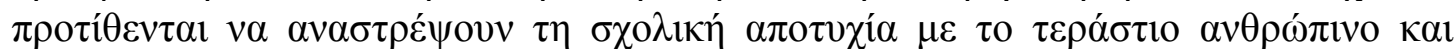

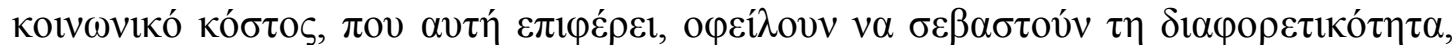

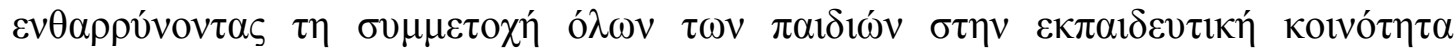
(Cummins, 2005).

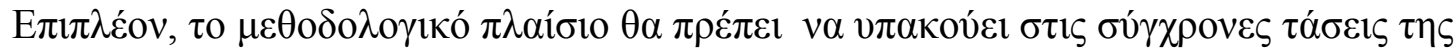

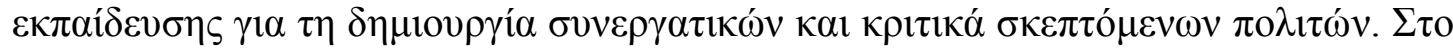

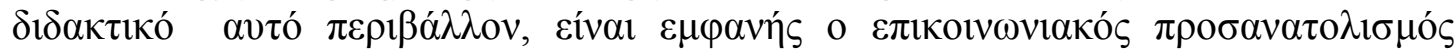

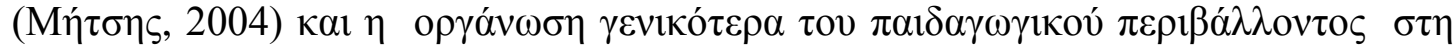

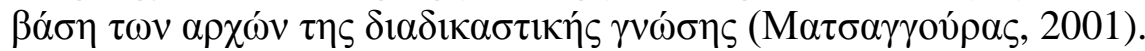

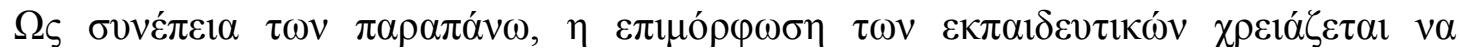

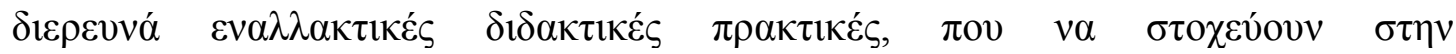

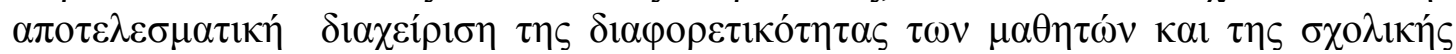

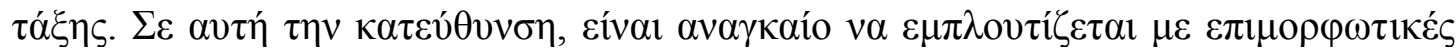

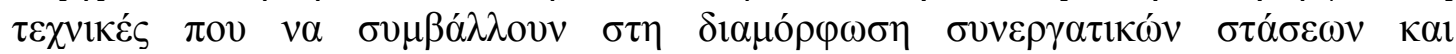

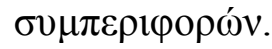

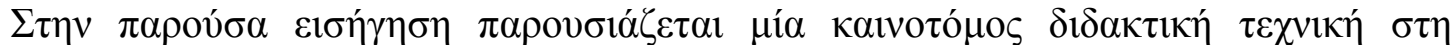

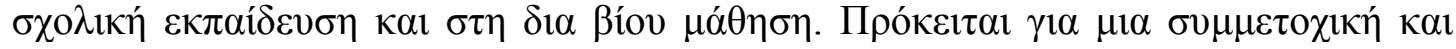

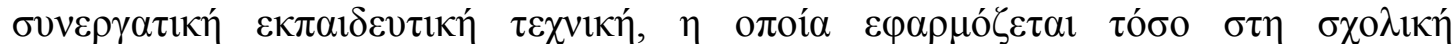

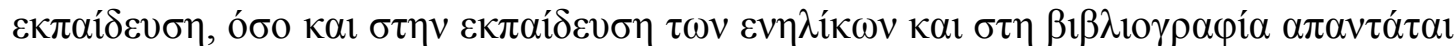

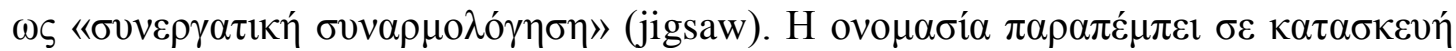

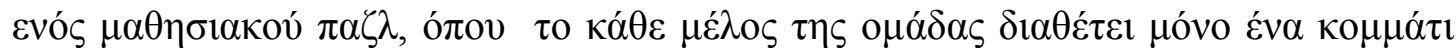

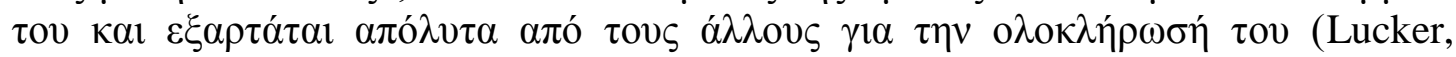
Rosenfield, Sikes \& Aronson, 1976).

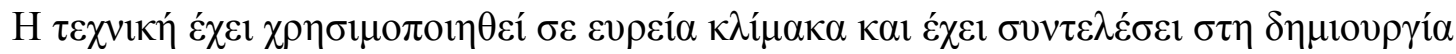

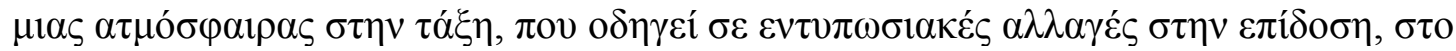

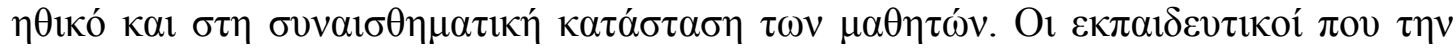

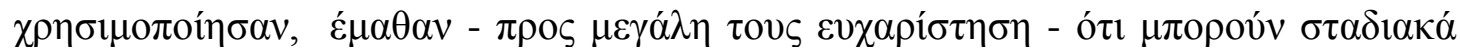

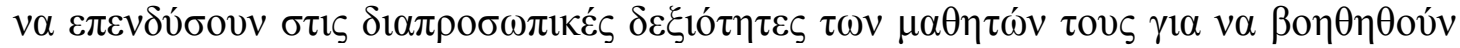

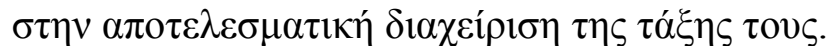

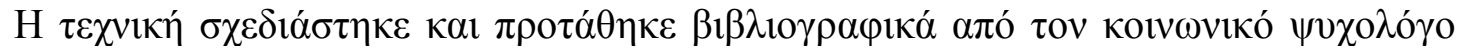

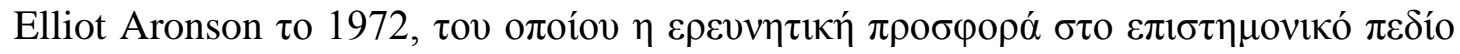

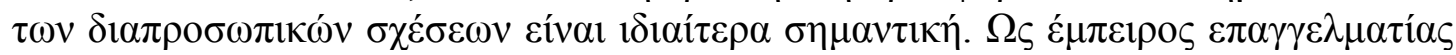

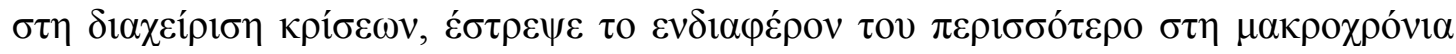

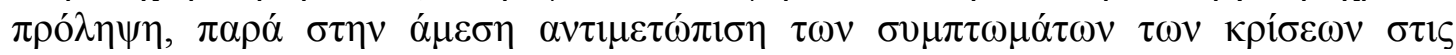

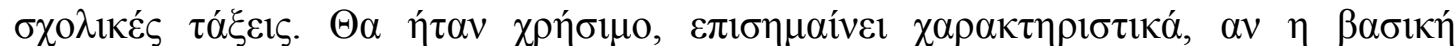

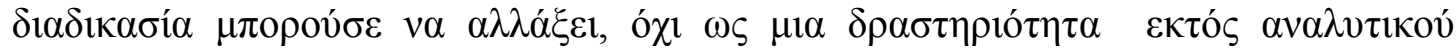

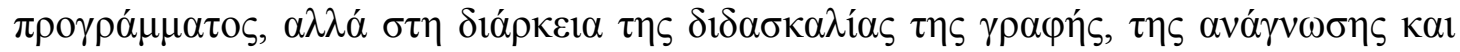




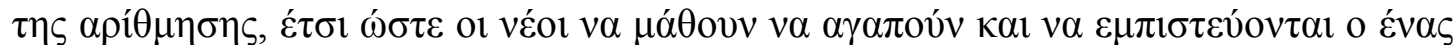

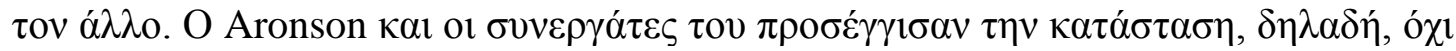

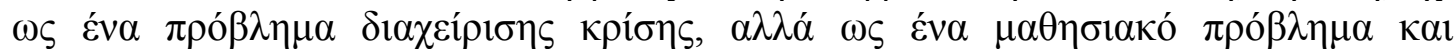

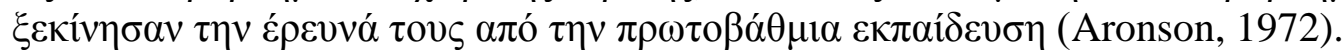

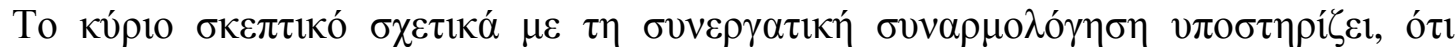

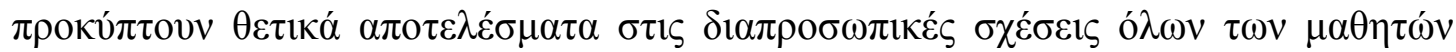

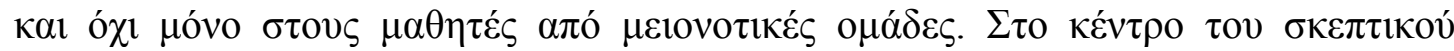

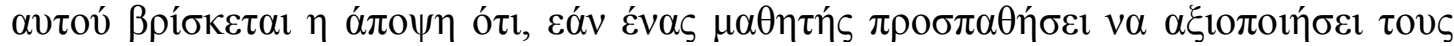

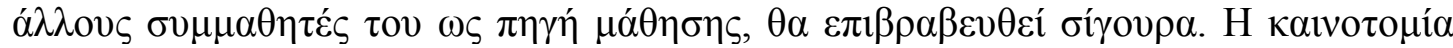

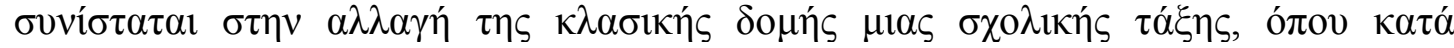

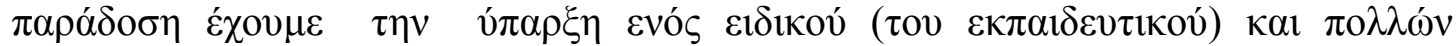

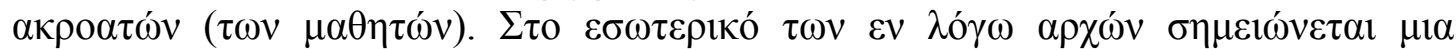

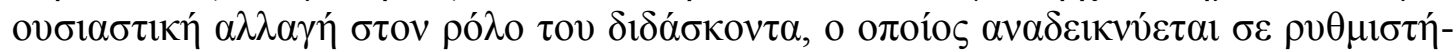

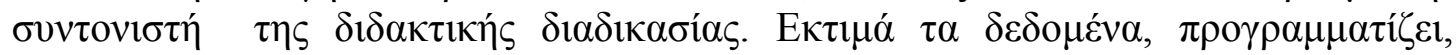

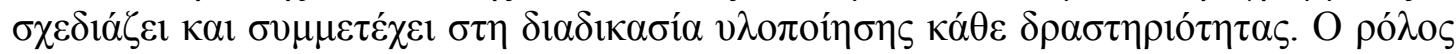

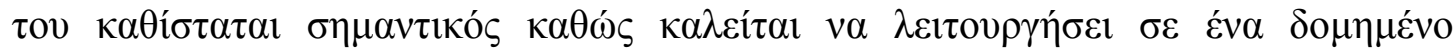

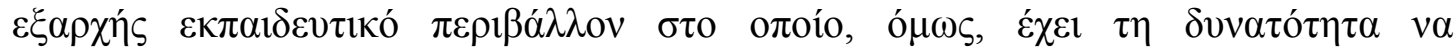

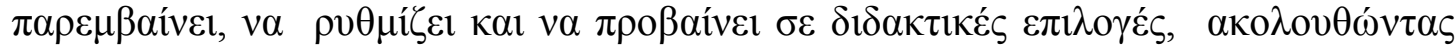

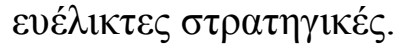

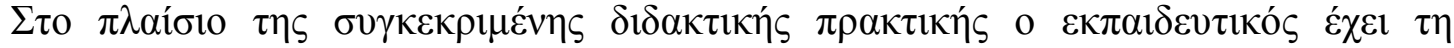
$\delta v v \alpha \tau o ́ \tau \eta \tau \alpha:$

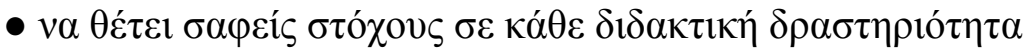

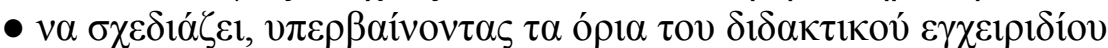

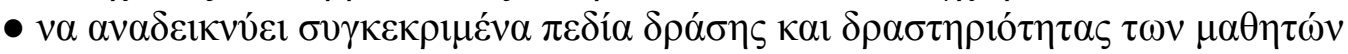

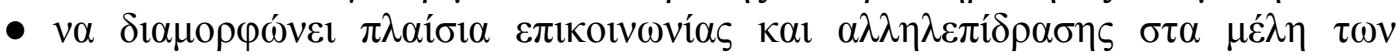

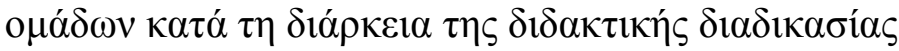

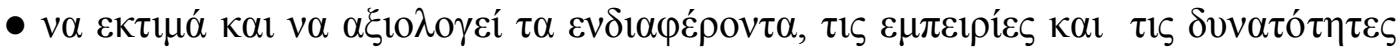
$\tau \omega v \mu \alpha \theta \eta \tau \omega ́ v$

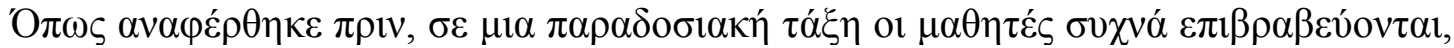

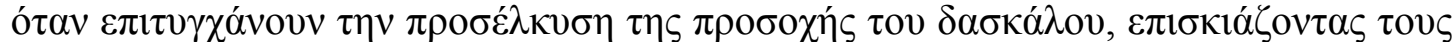

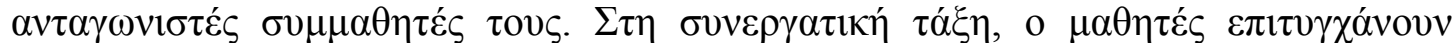

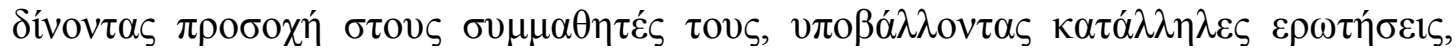

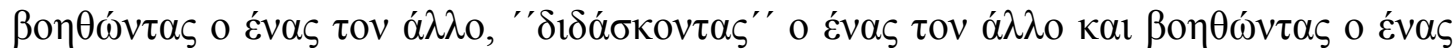

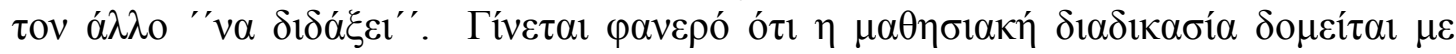

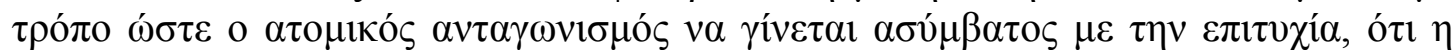

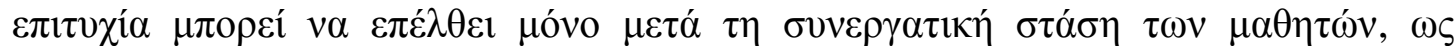

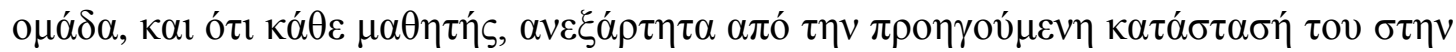

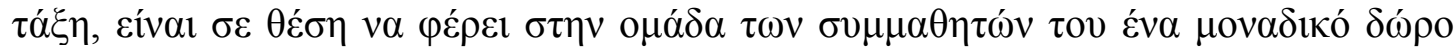

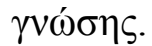

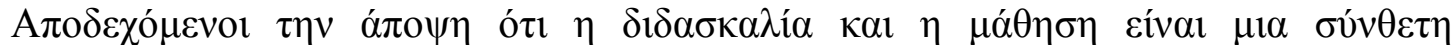

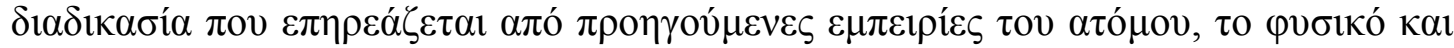

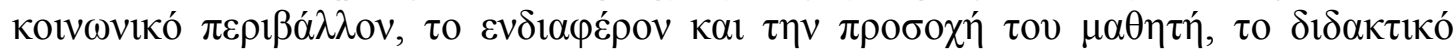

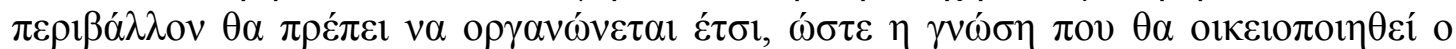

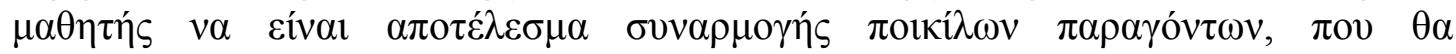

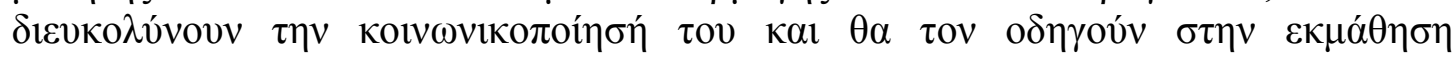

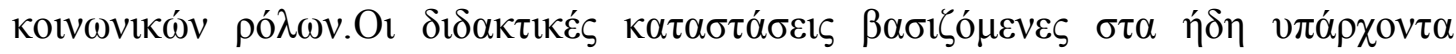

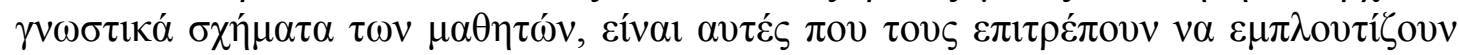

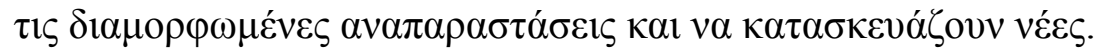

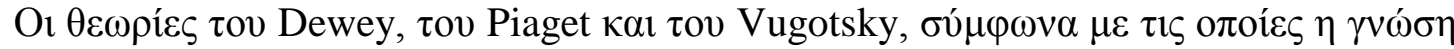

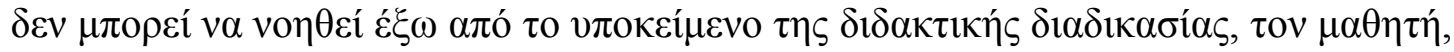




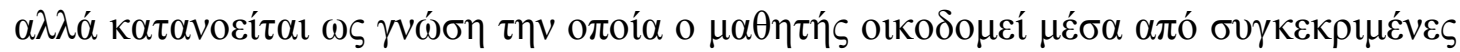

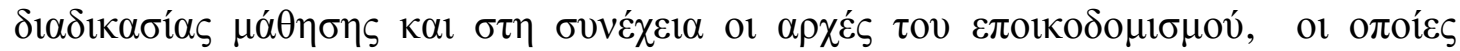

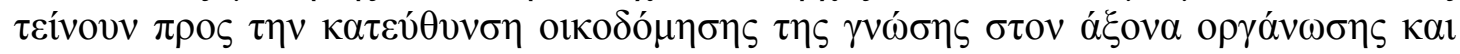

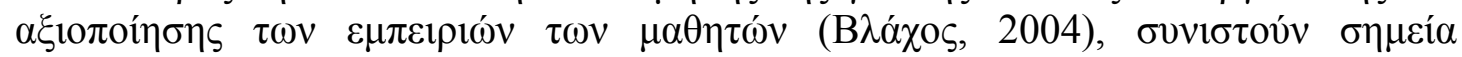

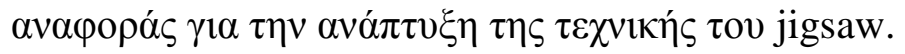

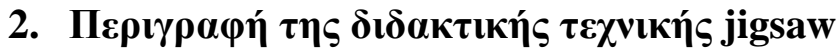

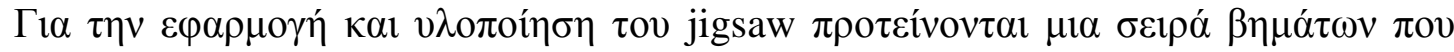

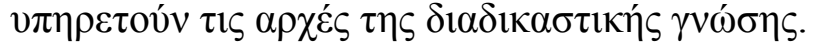

Bím 1
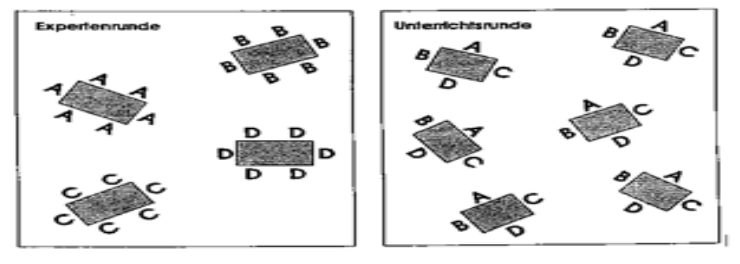

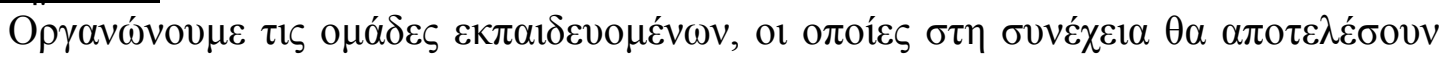

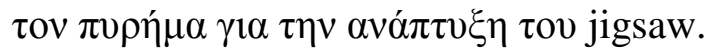

$\underline{B \eta} \mu \alpha 2$

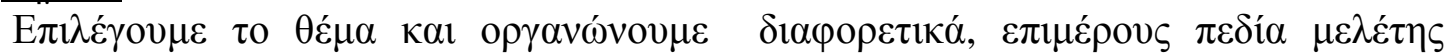

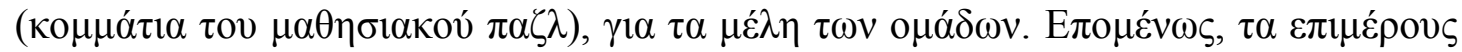

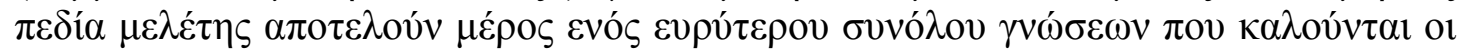

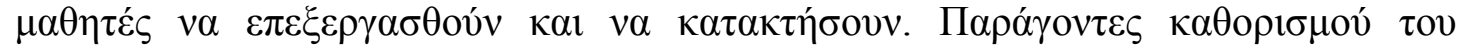

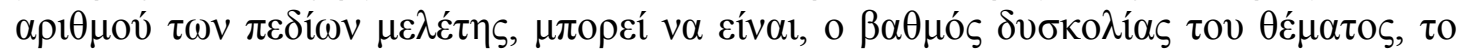

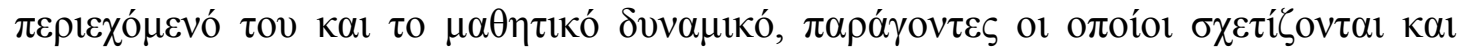

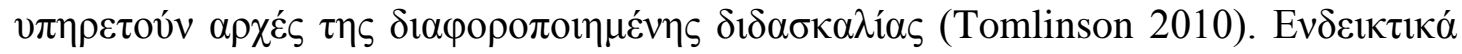

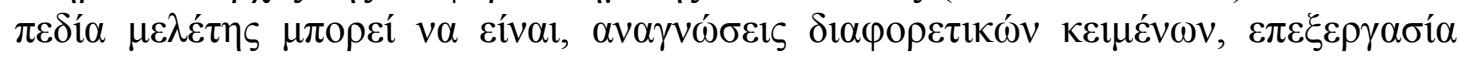

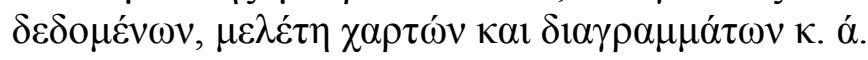

$\underline{B \eta \dot{\mu} \mu \alpha 3}$

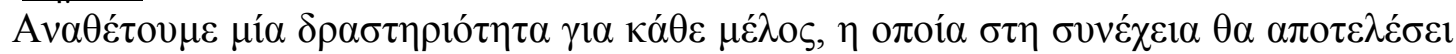

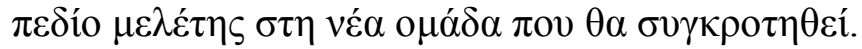

$\underline{B \eta} \mu \alpha 4$

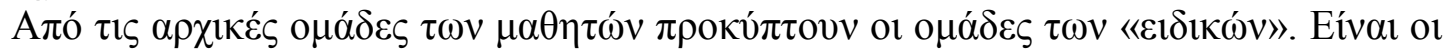

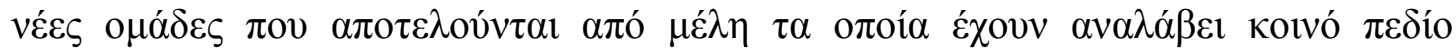

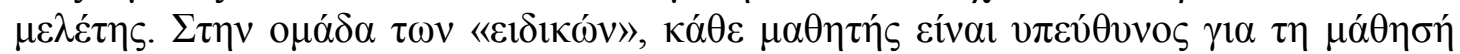

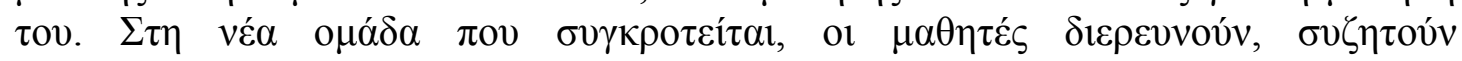

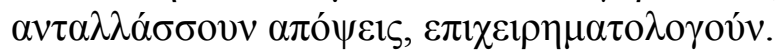

\section{$\underline{B \dot{\eta} \mu \alpha} 5$}

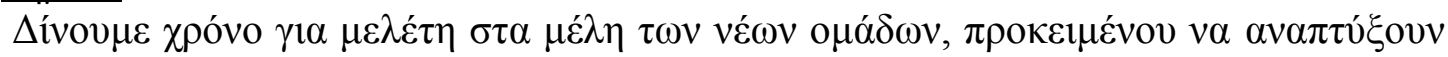

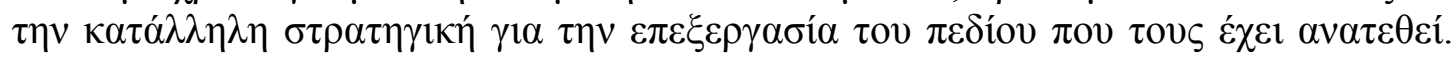

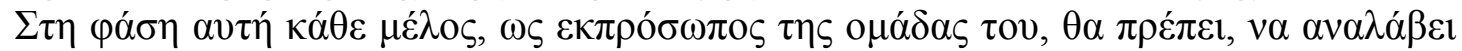

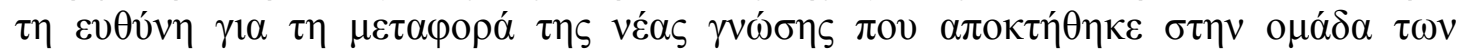

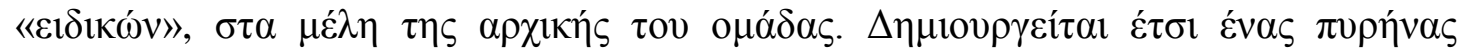

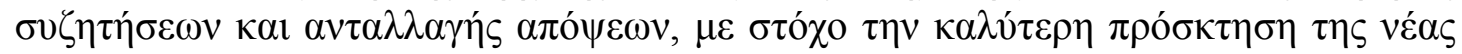

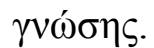

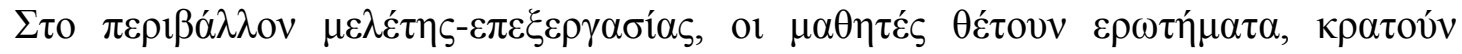

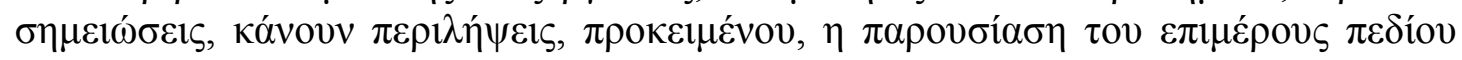

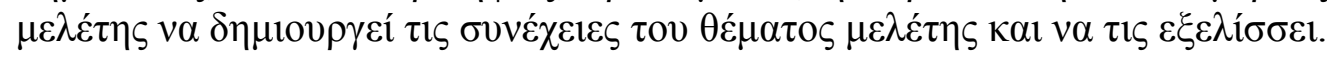

$\underline{B \eta \dot{\eta} \mu \alpha} 6$

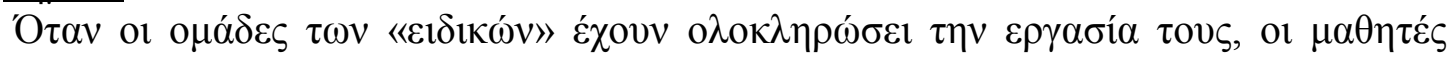

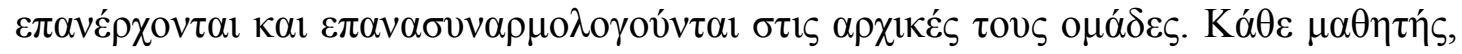

SECTION B: applications, experiences, good practices, descriptions and outlines, educational activities, issues for dialog and discussion 


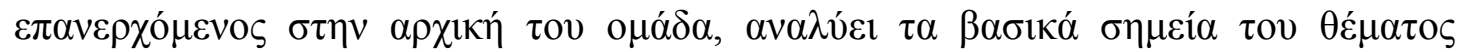

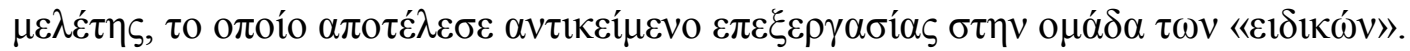

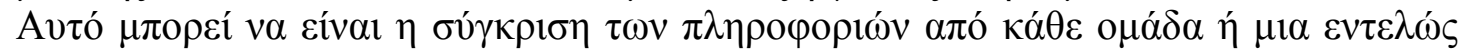

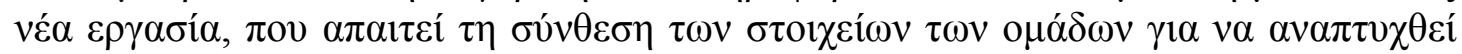

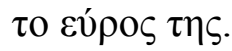

$\underline{B \eta} \mu \alpha 7$

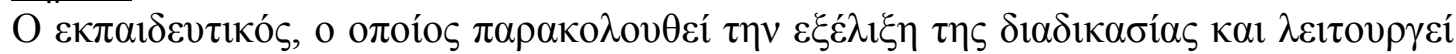

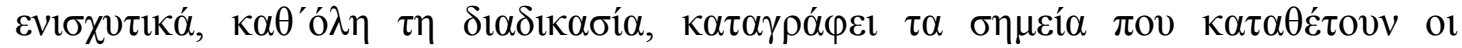

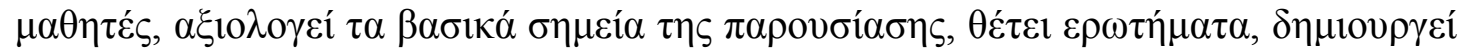

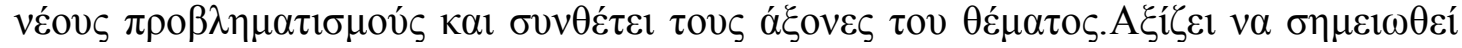

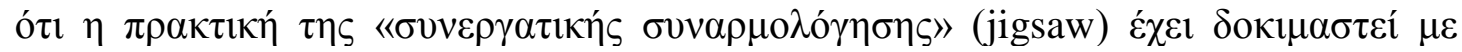

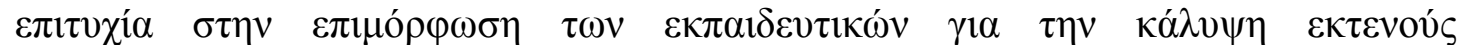

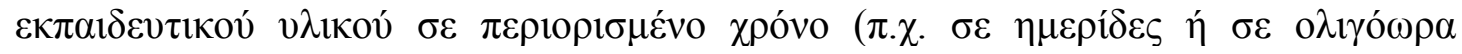

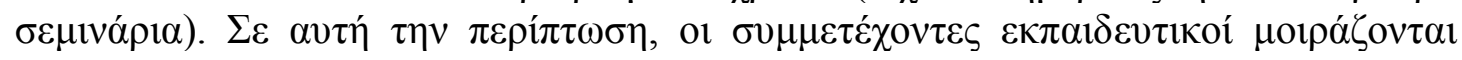

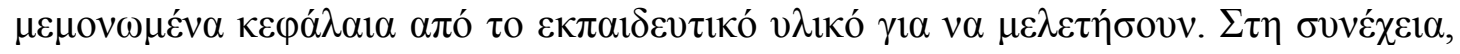

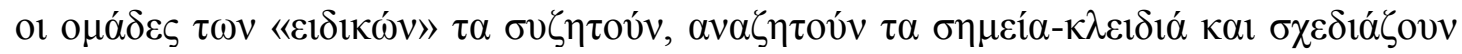

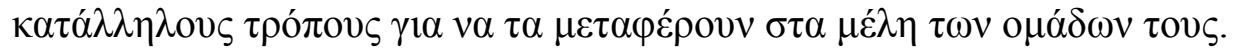

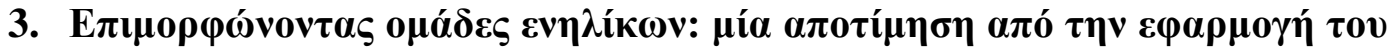 jigsaw}

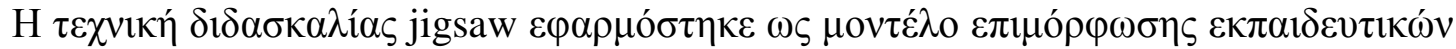

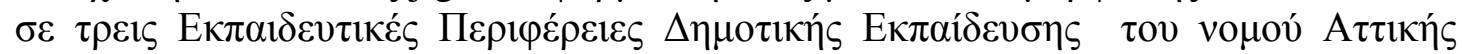

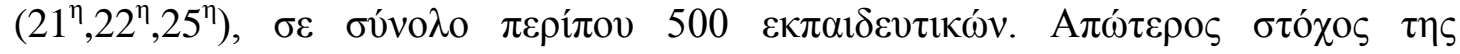

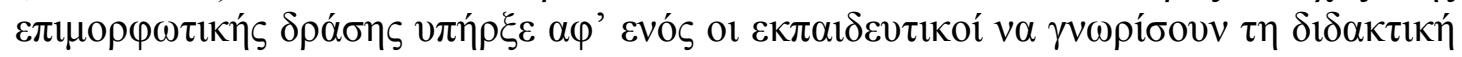

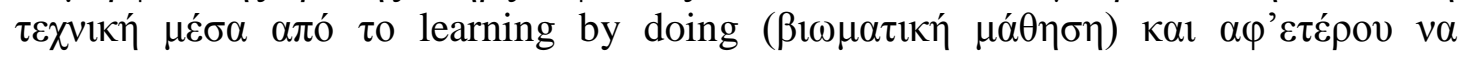

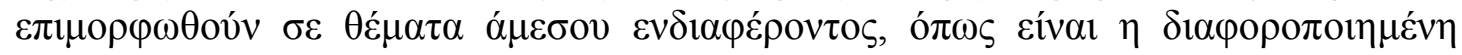
$\delta 1 \delta \alpha \sigma \kappa \alpha \lambda i \alpha, \quad \eta \pi \mathrm{o} \lambda \lambda \alpha \pi \lambda \eta \dot{~ v o \eta \mu о \sigma u ́ v \eta, ~} \eta \quad \delta 1 \alpha \pi \mathrm{o} \lambda \imath \tau 1 \sigma \mu 1 \kappa \eta \dot{~ \varepsilon \kappa \pi \alpha i ́ \delta \varepsilon v \sigma \eta} \kappa \alpha 1$ ol

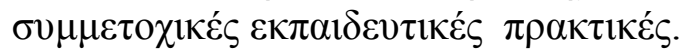

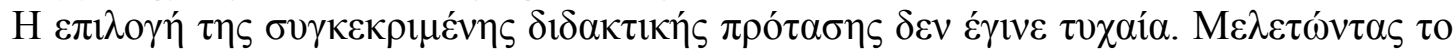

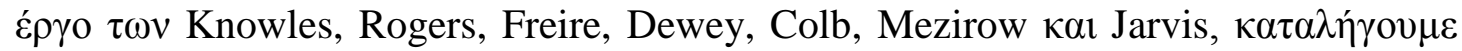

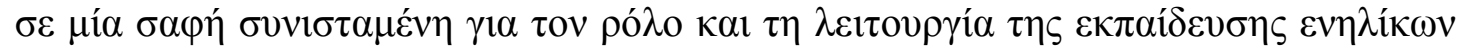

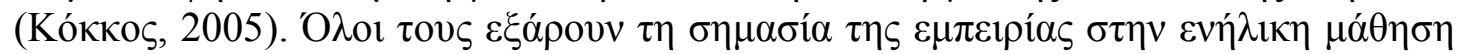

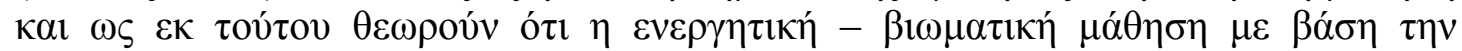

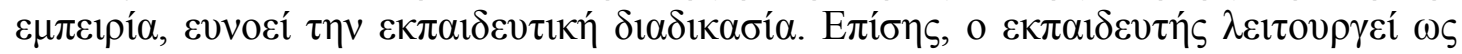

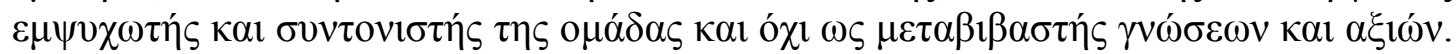

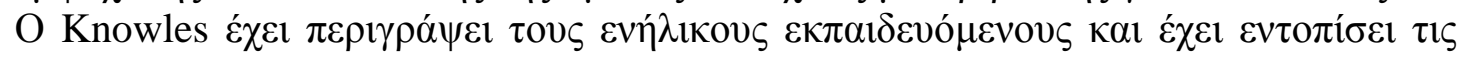

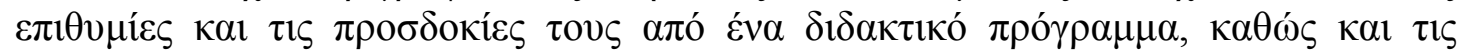

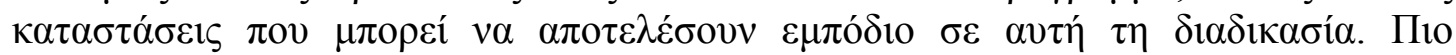

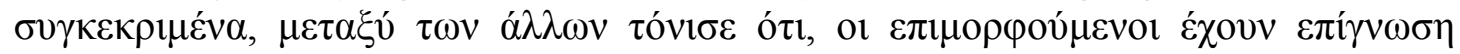

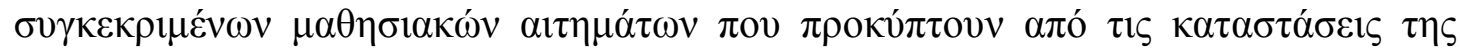

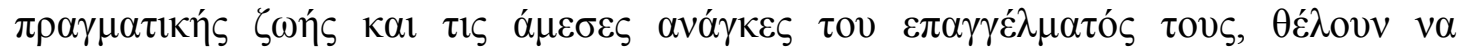

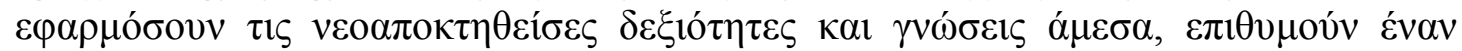

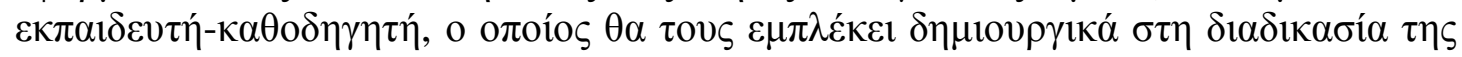

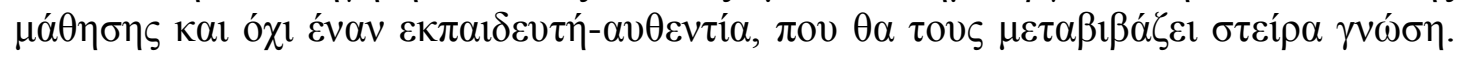

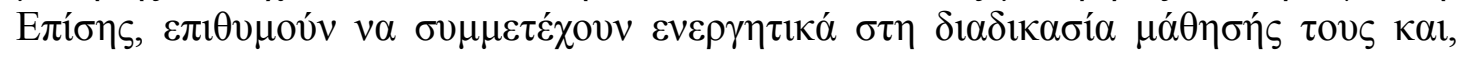

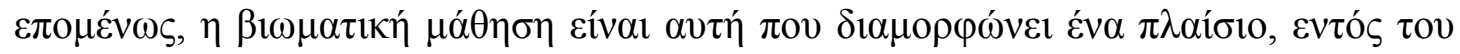

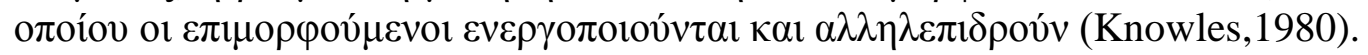

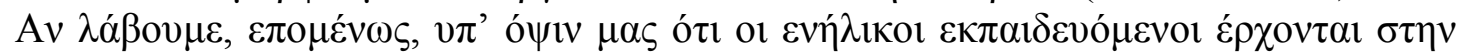

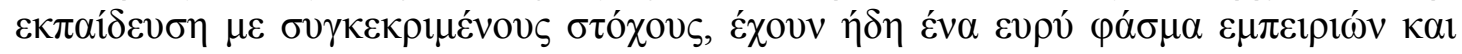

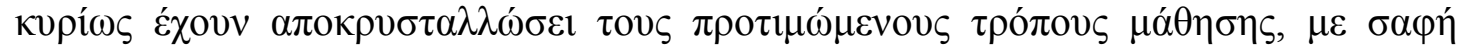




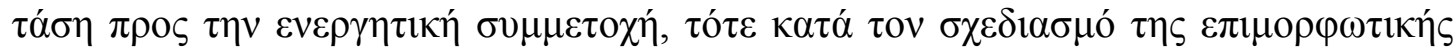

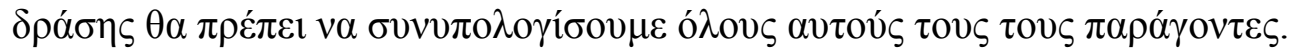

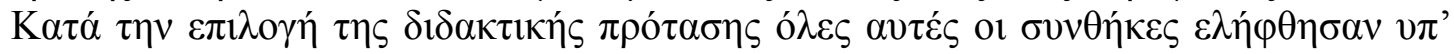

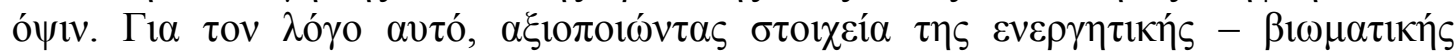

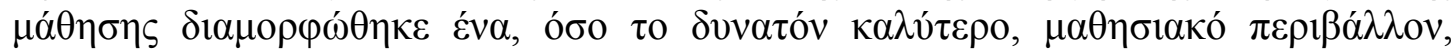

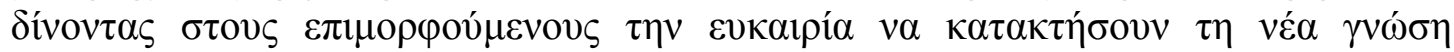

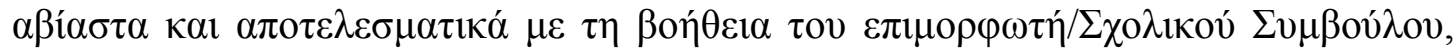

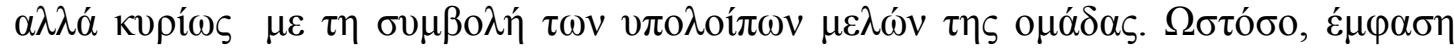

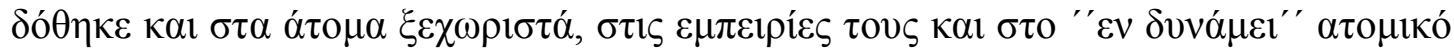

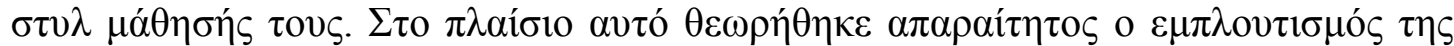

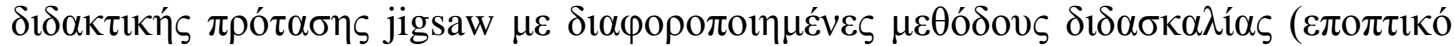

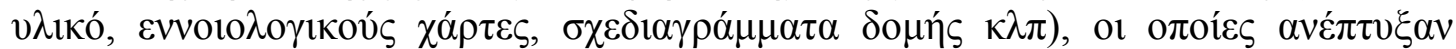

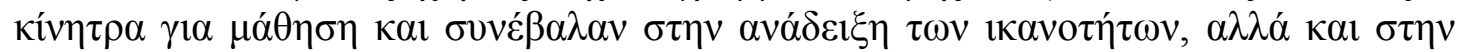

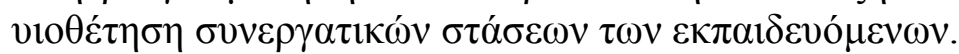

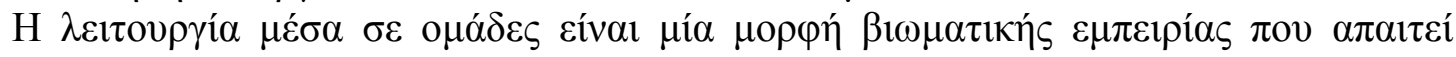

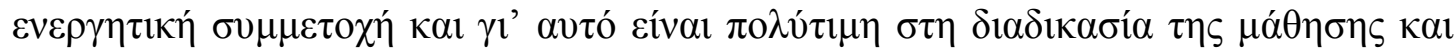

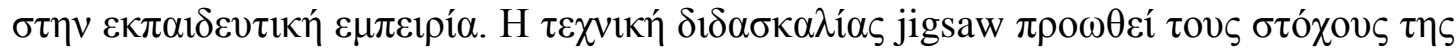

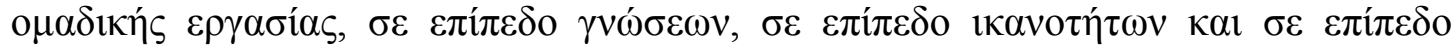

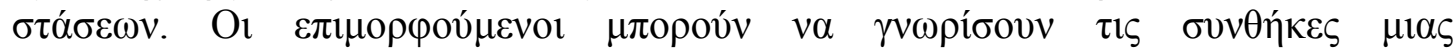

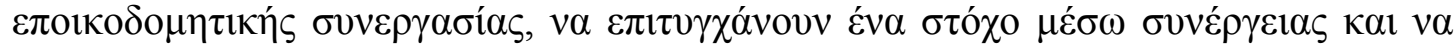

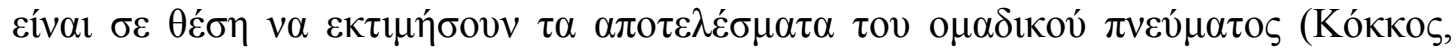
2007).

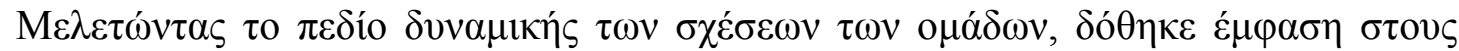

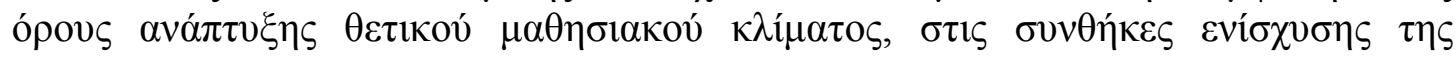

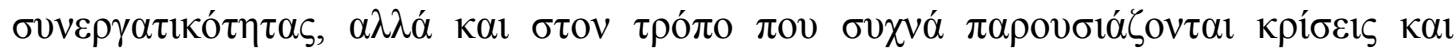

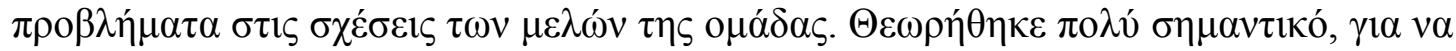

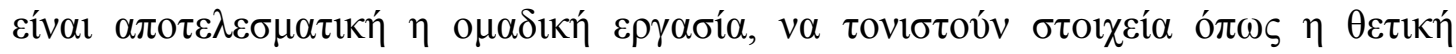

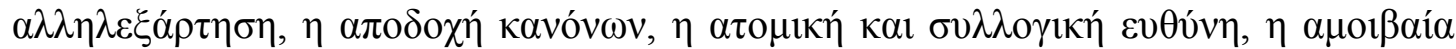

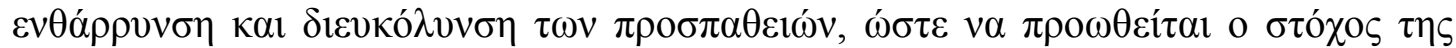

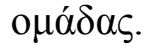

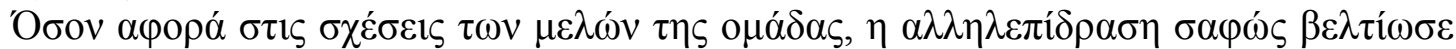

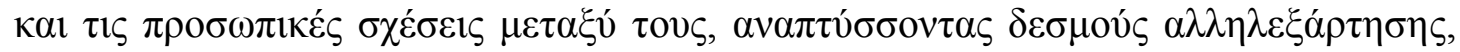

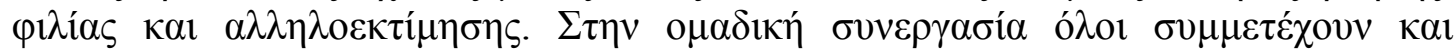

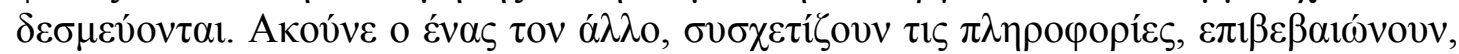

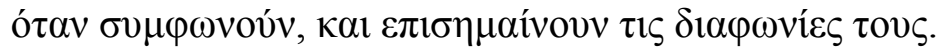

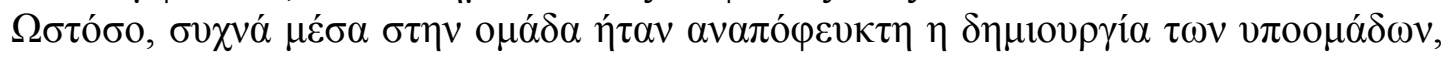

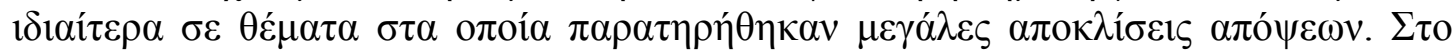

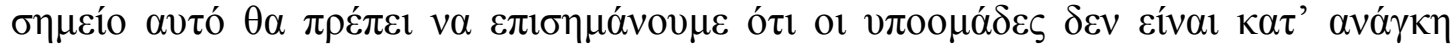

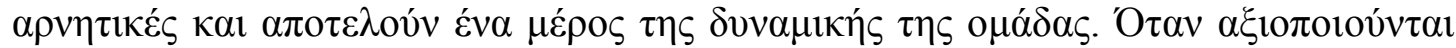

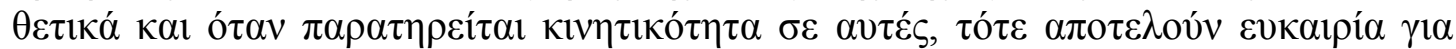

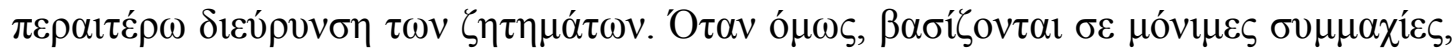

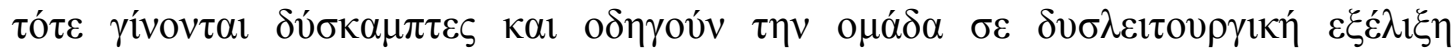
(Поде́ $\mu \eta-T o \delta o v ́ \lambda o v, 2007)$.

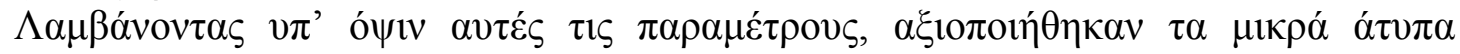

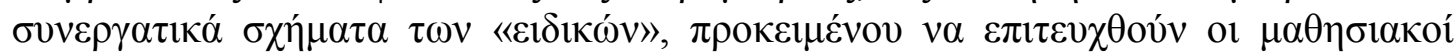

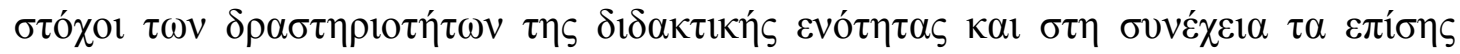

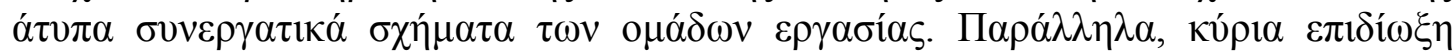

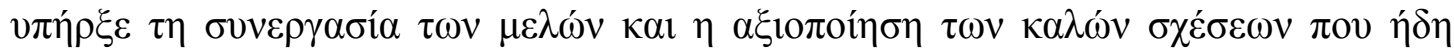

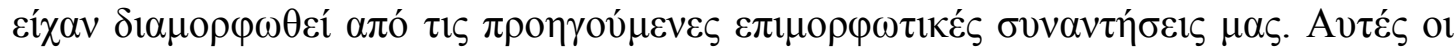

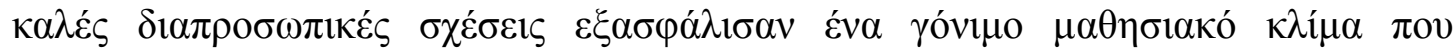




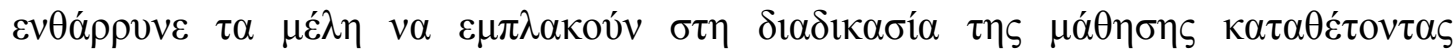

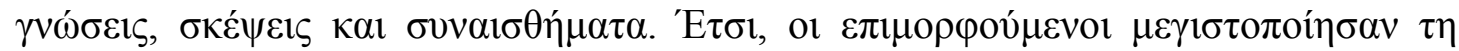

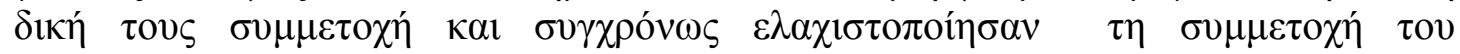

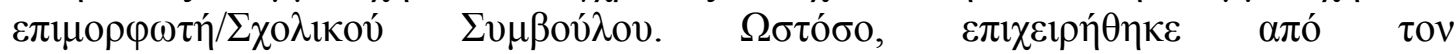

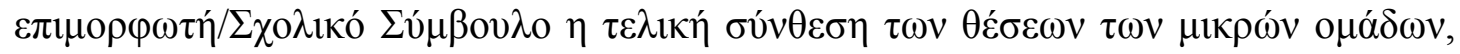

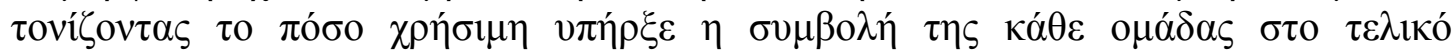

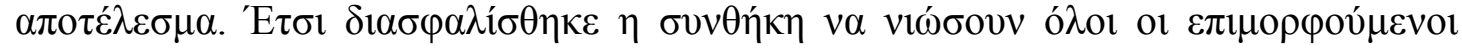

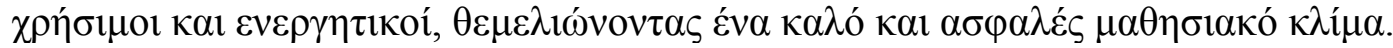

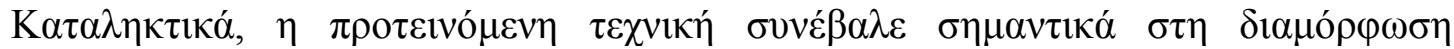

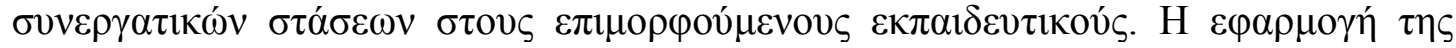

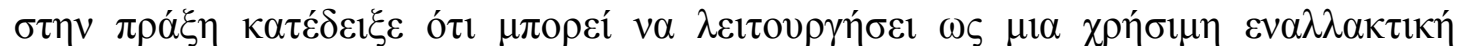

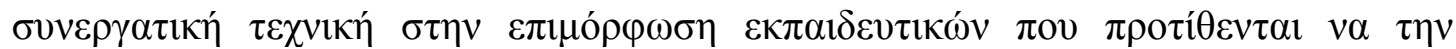

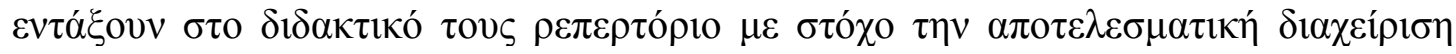

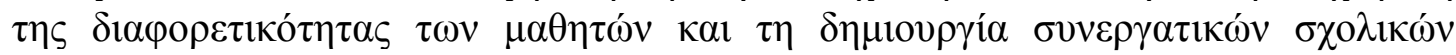

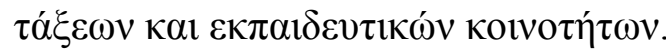

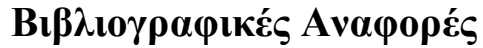

Aronson, E. (1972). The Social Animal. San Fransisco: W.H Freeman.

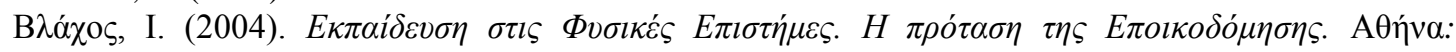

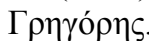

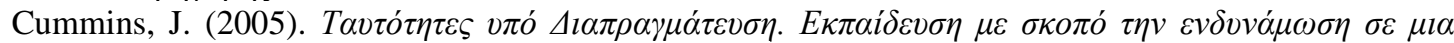

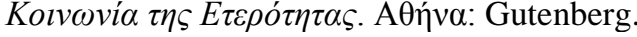

Groof, J. de., Lauwers, G. \& Dondelinger, G. (2002). Globalisation and Competition in Education. Oisterwijk: Wolf Legal Publishers.

Knowles, M. S. (1980). The modern practice of Adult Education: From Pedagogy to Andragogy. New York: Cambridge Books.

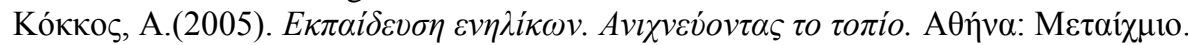

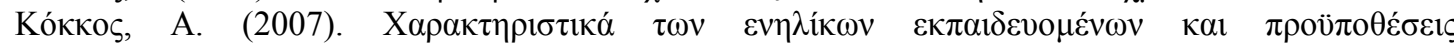

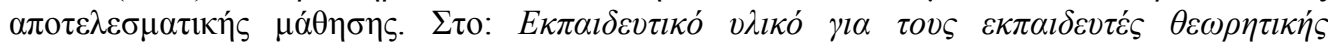

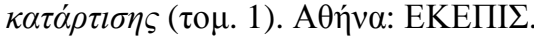

Lucker, G.W., Rosenfield, D., Sikes, J. \& Aronson, E. (1976). Performance in the interdepedent Classroom: A Field Study. American Educational Research Journal, Spring 1976, 13(2), 115 123.

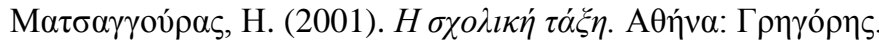

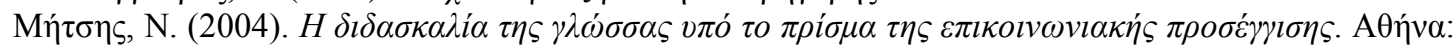
Gutenberg.

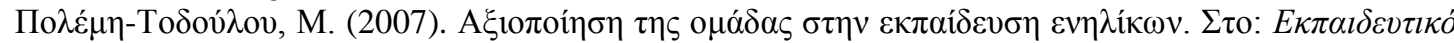

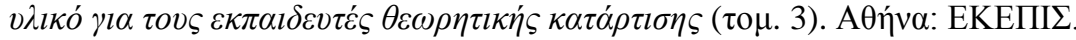

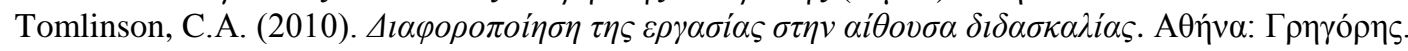

Vygotsky, L.S. (1978). Mind in Society. Cambridge, MA: Harvard University Press. 